\title{
A Compact Ultra-Wideband Bandpass Filter Based on Split-Mode Resonator
}

\author{
Tsung-Nan Kuo, Chi-Hsueh Wang, and Chun Hsiung Chen, Fellow, IEEE
}

\begin{abstract}
A compact ultra-wideband (UWB) bandpass filter is proposed based on the coplanar-waveguide (CPW) split-mode resonator. By suitably introducing a short-circuited stub to implement the shunt inductance between two quarter wavelength $(\lambda / 4) \mathrm{CPW}$ stepped-impedance resonators, a strong magnetic coupling may be realized so that a CPW split-mode resonator may be constructed. Moreover, by properly designing the dual-metal-plane structure, one may accomplish a microstrip-to-CPW feeding mechanism to provide strong enough capacitive coupling for bandwidth enhancement and also introduce an extra electric coupling between input and output ports so that two transmission zeros may be created for selectivity improvement. The implemented UWB filter shows a fractional bandwidth of $116 \%$ and two transmission zeros at 1.705 and $11.39 \mathrm{GHz}$. Good agreement between simulated and measured responses is observed.
\end{abstract}

Index Terms-Bandpass filter (BPF), coplanar waveguide (CPW), dual-plane structure, microstrip, split-mode resonator, ultra-wideband (UWB).

\section{INTRODUCTION}

$\mathbf{T}$ HE ultra-wideband (UWB) wireless communication technology has received great attention, especially after the Federal Communications Commission (FCC) decision to permit the unlicensed operation band from 3.1 to $10.6 \mathrm{GHz}$ in February 2002 [1]. The UWB systems have many attractive benefits such as transmitting higher data rates and requiring lower transmit power. However, for developing the UWB filters, there are a lot of challenges to meet the ultra-wide bandwidth requirement.

Over the past few years, the optimum distributed highpass filter prototype [2] was adopted to design the UWB filters. To reduce the circuit size, the technique of using folded lines and shared vias was employed to implement the UWB filters [3]. To further increase the selectivity, a cross coupling between feeding lines was added to create new pairs of transmission zeros [4]. Furthermore, to suppress the spurious response, electromagnetic bandgaps were adopted in [5]. However, these filters have the drawbacks of large circuit area and inconvenient via process.

Manuscript received May 24, 2007; revised August 1, 2007. This work was supported in part by the National Science Council of Taiwan, R.O.C., under Grant NSC 95-2752-E-002-001-PAE, Grant NSC 95-2221-E-002-196 and in part by the Excellent Research Projects of National Taiwan University, NTUERP-95R0062-AE00-00.

The authors are with the Department of Electrical Engineering and Graduate Institute of Communication Engineering, National Taiwan University, Taipei 106, Taiwan, R.O.C. (e-mail: chchen@ew.ee.ntu.edu.tw).

Color versions of one or more of the figures in this paper are available online at http://ieeexplore.ieee.org.

Digital Object Identifier 10.1109/LMWC.2007.910483
Multiple-mode resonator is another popular structure used for wideband filter design. In [6], the aperture compensation technology was proposed to increase the coupling between parallel-coupled lines. In [7], a five-pole UWB filter was built up based on a single triple-mode stepped-impedance resonator. In [8], the microstrip-to-CPW transition was used as a feeding structure. Since these filters are based on the half-wavelength $(\lambda / 2)$ multiple-mode resonators, they have large circuit sizes and poor selectivity.

Recently, dual-plane structures have become a competitive candidate for the filter design. In [9], the wideband filters using microstrip and CPW quarter-wavelength $(\lambda / 4)$ resonators were reported, however their bandwidths are not wide enough to meet the UWB requirement. In [10], the UWB filters based on the quasi-lumped-circuit elements were proposed. Although these filters possess good performances and compact sizes, their spurious passbands are close to the UWB passband.

In this letter, a compact UWB bandpass filter (BPF) based on the split-mode resonator is fabricated. The split-mode resonator is formed by introducing a CPW short-circuited stub into two $\lambda / 4$ CPW stepped-impedance resonators. By properly designing the split-mode resonator, the upper spurious passband may somewhat be suppressed. To accomplish a large capacitive coupling, the broadside-coupled microstrip-to-CPW transitions are adopted as the feeding structures for bandwidth enhancement. Moreover, an extra electric coupling between input and output ports is introduced to create two transmission zeros for selectivity improvement. Specifically, the implemented fourpole UWB filter has the merits of compact size, good insertion/return loss, improved selectivity, flat group delay, and better upper stopband response.

\section{FOUR-POLE UWB FILTER}

\section{A. Split-Mode Resonator}

Fig. 1 shows the proposed four-pole UWB filter using both microstrip and CPW structures. The proposed filter is based on the CPW split-mode resonator. As shown in Fig. 2(a), the splitmode resonator is composed of two $\lambda / 4$ stepped-impedance resonators and each resonator is resonated at the central frequency $f_{0}$. By introducing a shunt inductance to establish a strong magnetic coupling between two $\lambda / 4$ resonators, one may implement a split-mode resonator which has two split resonance frequencies at $f_{1}$ and $f_{2}$. Specifically, the larger the inductance, the stronger the coupling, the wider the split of two resonance frequencies, and the deeper the minimum in the $S_{21}$ curve. Furthermore, it should be noted that the electric lengths of these two resonators may be reduced by the introduction of inductance. 


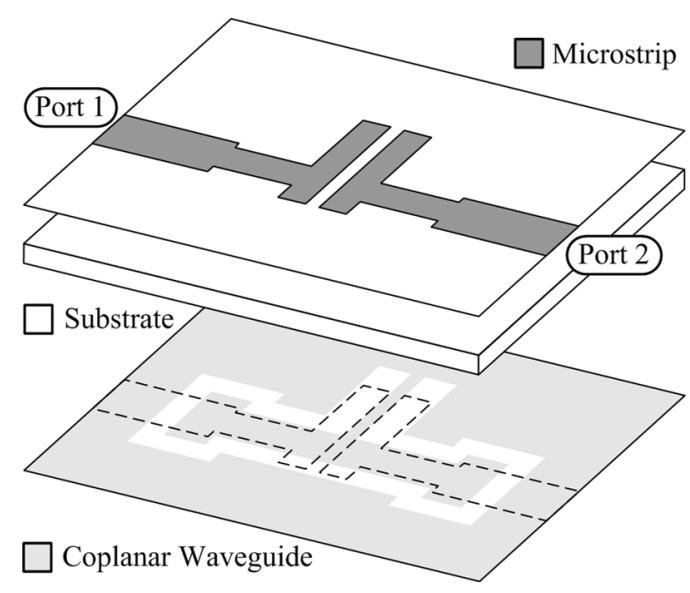

Fig. 1. Three-dimensional physical layout of the proposed four-pole UWB filter.

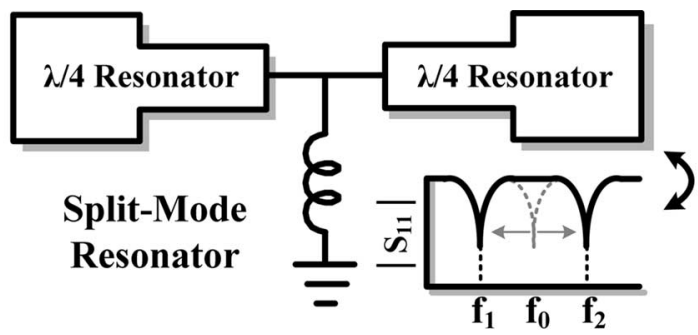

(a)

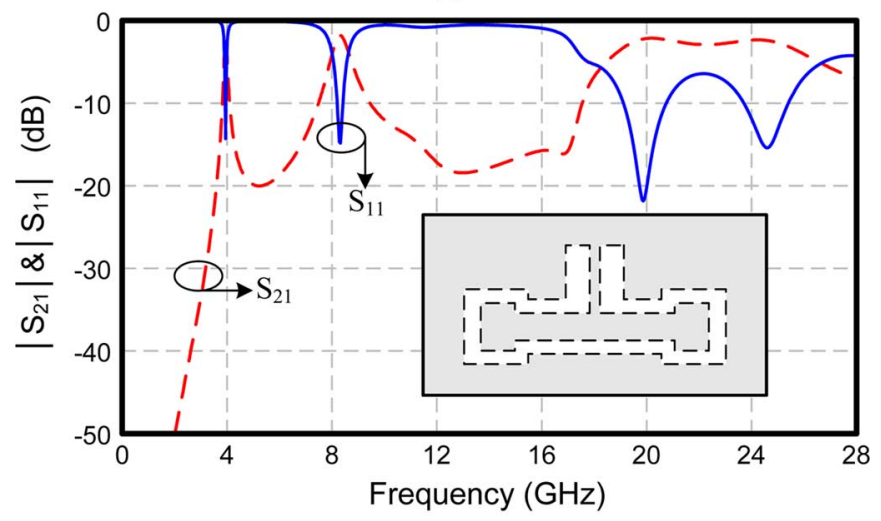

(b)

Fig. 2. Circuit structure and simulated responses of proposed CPW split-mode resonator.

To avoid using lumped-elements and metal vias, the CPW short-circuited stub is used to realize the shunt inductance. Under the ultra-wide bandwidth requirement, the CPW short-circuited stub must be designed so as to widely separate the two split resonance frequencies. By combining the two $\lambda / 4$ CPW resonators and the short-circuited stub, a CPW split-mode resonator is implemented. To investigate the resonance frequencies associated with the split-mode resonator, the simulator ADS Momentum is used. Fig. 2(b) shows the simulated $S$-parameters of the CPW split-mode resonator which is weakly coupled to the feeding structure. The simulated responses show that two split resonance frequencies $f_{1}$ and $f_{2}$ are observed at 4 and $8.3 \mathrm{GHz}$. Moreover, due to the use

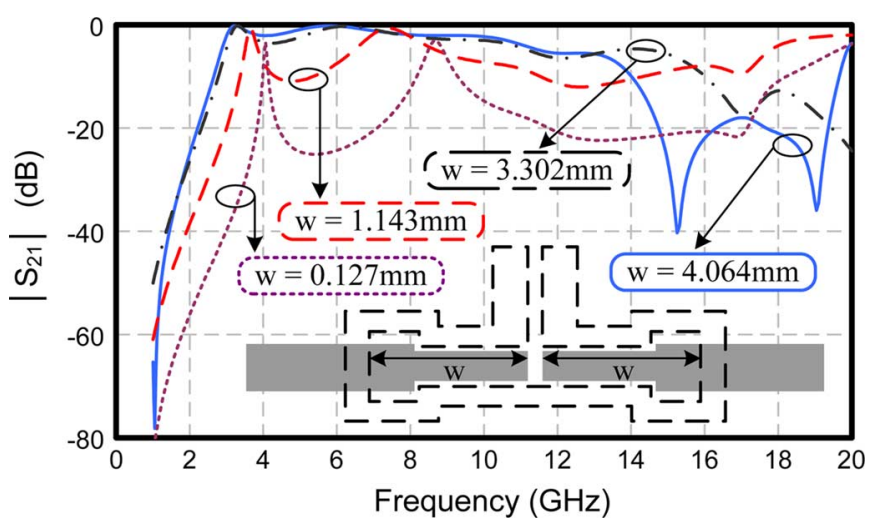

Fig. 3. Simulated frequency responses of the filter with transitions of various microstrip length $w$.

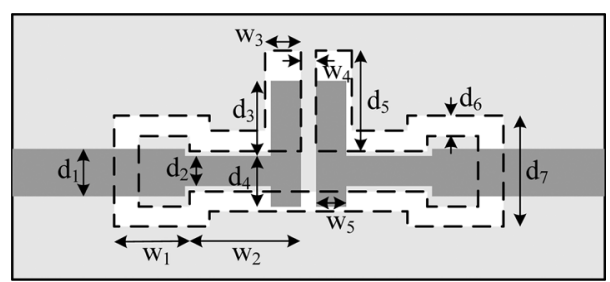

Fig. 4. Top-/bottom-layer circuit layouts of the proposed four-pole UWB filter. $\left(w_{1}=1.905 \mathrm{~mm}, w_{2}=2.794 \mathrm{~mm}, w_{3}=0.889 \mathrm{~mm}, w_{4}=0.381 \mathrm{~mm}, w_{5}=\right.$ $0.762 \mathrm{~mm}, d_{1}=1.194 \mathrm{~mm}, d_{2}=0.762 \mathrm{~mm}, d 3=1.905 \mathrm{~mm}, d_{4}=1.27 \mathrm{~mm}$, $d_{5}=2.54 \mathrm{~mm}, d_{6}=0.508 \mathrm{~mm}$, and $d_{7}=2.794 \mathrm{~mm}$ ).

of $\lambda / 4$ stepped-impedance resonator which makes the spurious passband beyond the triple center frequency, higher unwanted resonances appear around 19.87 and $24.61 \mathrm{GHz}$. Note that these higher unwanted resonances would dominate the spurious passband of the proposed UWB filter.

\section{B. Transition and Extra Electric Coupling}

Strong couplings in input and output stages are required in designing a broadband filter. In this study, to fit in with the UWB requirement, the broadside-coupled microstrip-to-CPW transitions are adopted as the input/output feeders so as to provide strong enough capacitive couplings for bandwidth enhancement. Here, the simulated responses associated with the transition structure are illustrated in Fig. 3. By increasing the microstrip length w (Fig. 3) to increase the overlap area between microstrip and CPW, the insertion loss around the minimum is reduced and becomes smooth.

Based on the magnetic coupling provided by the split-mode resonator, it is easy to create two transmission zeros at the lower and upper stopbands by introducing an extra electric coupling between the input and output ports. Taking advantages of the dual-metal-plane layout, the required electric coupling may be implemented by introducing the microstrip stubs of length $\left(d_{3}+\right.$ $\left.d_{4}\right)$ as shown in Fig. 4. Fig. 4 shows the top-/bottom-layer circuit layouts of the proposed UWB filter. The effect of adjusting the microstrip stub length $d_{3}$, which controls the electric coupling level, is illustrated in Fig. 5. Specifically, the lower and upper transmission zeros will move close to the passband edge as the stub length $d_{3}$ is increased. 


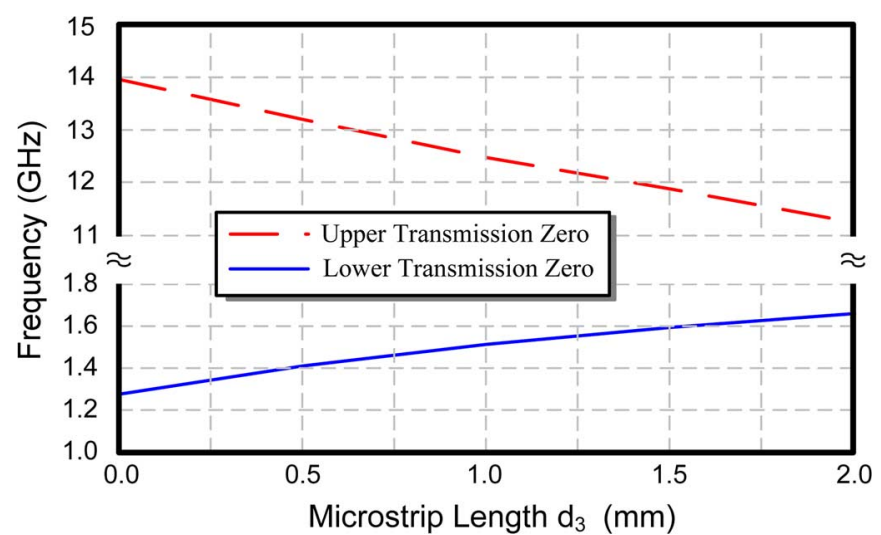

Fig. 5. Curves to relate the transmission-zero frequencies to the values of the microstrip length $d_{3}$ specified in Fig. 4.

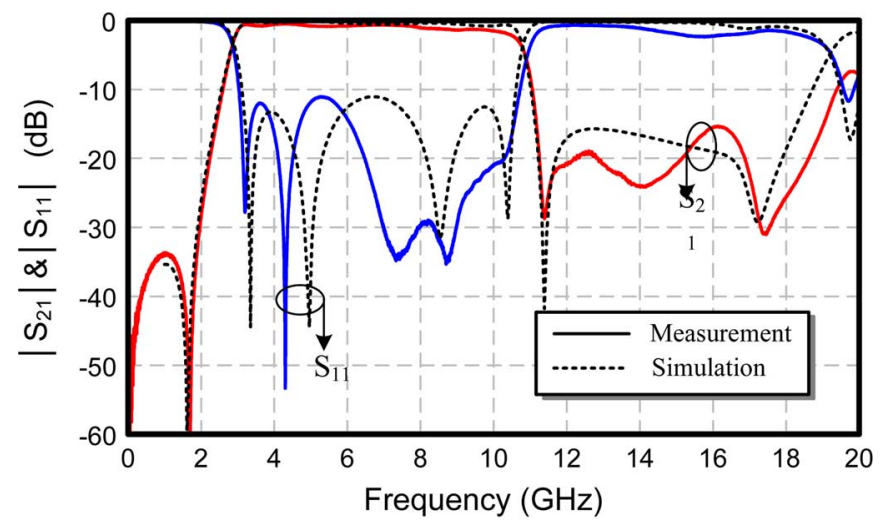

(a)

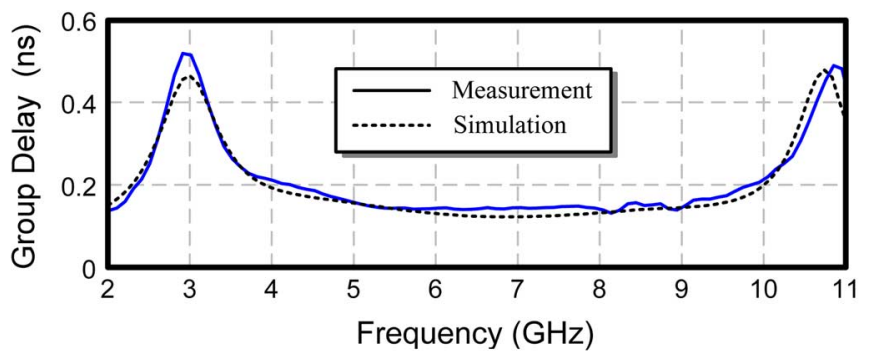

(b)

Fig. 6. Measured and simulated results of the proposed four-pole UWB filter (Fig. 4). (a) Scattering parameters. (b) Group delay.

\section{Measurement and Simulation Results}

Fig. 6(a) shows the measured and fullwave simulated frequency responses of the four-pole UWB filter (see Fig. 4), which is fabricated on the Rogers $\mathrm{RO} 4003 \mathrm{C}$ substrate with $\varepsilon_{r}=3.38$, $\tan \delta=0.002$, and thickness $h=0.508 \mathrm{~mm}$. The measured center frequency is at $6.8 \mathrm{GHz}$ and the measured $3-\mathrm{dB}$ fractional bandwidth is $116 \%$. The implemented filter has an insertion loss better than $0.53 \mathrm{~dB}$ and a return loss greater than $11 \mathrm{~dB}$ within the passband. Two transmission zeros are found at 1.705 and $11.39 \mathrm{GHz}$ and no spurious passband is observed from 12 to
$19 \mathrm{GHz}$. Moreover, the simulated return loss shows that the proposed filter has four poles. The extra two poles are contributed by the input and output feeding structures [6].

Fig. 6(b) exhibits a flat group delay response below $0.51 \mathrm{~ns}$ over the whole passband. The four-pole UWB filter has a compact dimension of $9.625 \mathrm{~mm} \times 4.375 \mathrm{~mm}$, which is approximately $0.36 \lambda_{g} \times 0.167 \lambda_{g}$, where $\lambda_{g}$ is the guided wavelength of microstrip structure at the center frequency of $6.8 \mathrm{GHz}$.

\section{CONCLUSION}

In this letter, a four-pole UWB filter based on the CPW split-mode resonator has been realized and carefully examined. The split-mode resonator is implemented by the CPW structure and has two split resonance frequencies in the UWB passband. By the dual-metal-plane layout, the broadside-coupled microstrip-to-CPW transitions and extra electric coupling may be incorporated in the filter design so that a compact UWB filter may be implemented with two transmission zeros created for selectivity improvement. Note that the implemented UWB filter has no spurious passband from 12 to $19 \mathrm{GHz}$, since the spurious harmonics of split-mode resonator have moved up to the higher frequencies. Compared to our previous work [10], this four-pole UWB filter has better performance than the three-pole one and more compact size than the five-pole one. The implemented filter has the merits of compact size and better performance when compared with the previously fabricated UWB filters.

\section{REFERENCES}

[1] Federal Communications Commission, Revision of Part 15 of the Commission's Rules Regarding Ultra-Wideband Transmission Systems FCC, Tech. Rep., ET-Docket FCC02-48, Feb. 2002, pp. 98-153.

[2] J. S. Hong and M. J. Lancaster, Microstrip Bandpass Filters for RF/Microwave Applications. New York: Wiley, 2001, ch. 6.

[3] W. T. Wong, Y. S. Lin, C. H. Wang, and C. H. Chen, "Highly selective microstrip bandpass filters for ultra-wideband (UWB) applications," in Proc. Asia-Pacific Microw. Conf, Nov. 2005, pp. 2850-2853.

[4] H. Shaman and J. S. Hong, "A novel ultra-wideband vandpass filter with pairs of transmission zeros," IEEE Microw. Wireless Compon. Lett., vol. 17, no. 2, pp. 121-123, Feb. 2007.

[5] J. G. Garcia, J. Bonache, and F. Martin, "Application of electromagnetic bandgaps to the design of ultra-wide bandpass filters with good out-of-band performance," IEEE Trans. Microw. Theory Tech, vol. 54, no. 12, pp. 4136-4140, Dec. 2006.

[6] L. Zhu, H. Bu, and K. Wu, "Aperture compensation technique for innovative design of ultra-broadband microstrip bandpass filter," in IEEE MTT-S Int. Dig., 2000, pp. 315-318.

[7] L. Zhu, S. Sun, and W. Menzel, "Ultra-wideband (UWB) bandpass filters using multiple-mode resonator," IEEE Microw. Wireless Compon. Lett., vol. 15, no. 11, pp. 796-798, Nov. 2005.

[8] H. Wang, L. Zhu, and W. Menzel, "Ultra-wideband bandpass filters with hybrid microstrip/CPW structure," IEEE Microw. Wireless Compon. Lett., vol. 15, no. 12, pp. 844-846, Dec. 2005.

[9] T. N. Kuo, S. C. Lin, C. H. Wang, and C. H. Chen, "Compact bandpass filters based on dual-plane microstrip/coplanar-waveguide structure with quarter-wavelength resonators," IEEE Microw. Wireless Compon. Lett., vol. 17, no. 3, pp. 178-180, Mar. 2007.

[10] T. N. Kuo, S. C. Lin, C. H. Wang, and C. H. Chen, "Compact ultrawideband bandpass filters using composite microstrip-coplanar-waveguide structure," IEEE Trans. Microw. Theory Tech., vol. 54, no. 10, pp. 3772-3778, Oct. 2006. 